\title{
The discovery of elements 107 to 112
}

\author{
Sigurd Hofmann $n^{1,2, a}$ \\ ${ }^{1}$ GSI Helmholtzzentrum für Schwerionenforschung, 64291 Darmstadt, Germany \\ ${ }^{2}$ Institut für Physik, Goethe-Universität Frankfurt, 60438 Frankfurt, Germany
}

\begin{abstract}
In the 1960s, theoretical concepts prepared the path to nuclear matter with proton and neutron numbers far beyond the nuclei known at that time. The new laboratory GSI was founded for research on reactions with heavy ions, in particular those for production of the predicted superheavy nuclei. In this contribution it is presented how the interaction between experiment and theory resulted in a continuous improvement of the experimental set-ups on the one hand, and of the knowledge of the processes during the nuclear reaction and of the properties of the produced nuclei on the other hand. In the course of this work six new elements from 107 to 112 were produced and identified. An overview of the present status of experimental results and a comparison with theoretical interpretations is given.
\end{abstract}

\section{Expectations of research on super-heavy nuclei in the $1960 \mathrm{~s}$}

The 1960s were a trailblazing decade for research on super-heavy nuclei (SHN). Maria Goeppert Mayer, in her Nobel Lecture in 1963, presented an extended shell model for the nucleus reaching up to proton number $Z=184$ [1]. Using the right interaction between spin and angular momentum of the nucleons, the known magic numbers appeared as shell gaps up to $Z=82$ for the protons and $N=126$ for the neutrons. New magic numbers were predicted at $Z=126$ and 184, the latter value as a new magic number also for the neutrons.

In the middle of the 1960s, the concept of the macroscopic-microscopic (MM) model for calculating binding energies of nuclei was invented by V.M. Strutinsky [2]. In this model a microscopic shell-correction energy (SCE) which considers the shell structure of the nuclei, is added to the macroscopic part derived from the liquid drop model. Positive or negative SCE values decrease or increase the macroscopic part of the binding energy, respectively.

Using this method a number of the measured phenomena could be naturally explained. In particular, it became possible to calculate the binding energy of a heavy fissioning nucleus as function of deformation and thus to determine the fission barrier. Partial fission half-lives were calculated as a quantum mechanical tunneling process through the so determined fission barrier. The calculations revealed the existence of so called 'islands of stability' located in the vicinity of shell closures for both protons and neutrons far beyond the known nuclei. In addition, instead of the earlier magic number 126 for the protons, a new number 114 was calculated [3-13].

An important result which could be explained quite naturally applying the Strutinsky method for calculation of the structure of the fission barrier were the fission isomers

\footnotetext{
a e-mail: S.Hofmann@gsi.de
} 
discovered by S.M. Polikanov et al. in 1962 [14]. They gain their stability from a second minimum in the fission barrier at large deformation.

Great excitement among physicists and chemists created a prediction by T. Sikkeland in 1966. Based on high fission barriers of up to $12.1 \mathrm{MeV}$ for the doubly magic nucleus $Z=126$ and $N=184$, probability ratios for neutron emission versus fission $\left(\Gamma_{n} / \Gamma_{f}\right)$ of up to 100 were calculated for compound nuclei $(\mathrm{CN})$ of isotopes of element 126 produced in fusion reactions of heavy beam and target nuclei [15]. Such high survival probabilities resulted in high cross-sections for synthesis of SHN of up to $100 \mathrm{mb}$. The heaviest nuclei investigated at that time were produced only on a level of $1 \mathrm{nb}$. These were isotopes of element 105 studied in fusion reactions of light beams with targets of ${ }^{243} \mathrm{Am}$ in Dubna [16] and ${ }^{249} \mathrm{Cf}$ in Berkeley [17].

On that background, the Gesellschaft für Schwerionenforschung (GSI) was founded in 1969 for research on interactions of heavy ions and nuclei with matter with the linear accelerator UNILAC as the central device [18]. One focus of the research program was the synthesis of SHN and the study of their radioactive decay modes.

\section{The velocity filter SHIP and early experiments}

The main instrument planned for study of SHN in fusion reactions was the velocity filter SHIP. The design was suggested by the research group of H. Ewald and G. Münzenberg from the Justus-Liebig-Universität in Gießen [19]. A schematic three dimensional view of SHIP is shown in Fig. 1. The greatest progress was expected from the short separation time of the fusion products determined by the flight time of the ions through the separator of $1-2 \mu \mathrm{s}$.

The velocity filter was the first major instrument ready when first beams were delivered from the UNILAC at the beginning of 1976. Already in May of that year first spectra from our study of the test reaction ${ }^{40} \mathrm{Ar}+{ }^{144} \mathrm{Sm}$ were presented by P. Armbruster at the conference on Nuclei far from Stability in Cargèse [20]. At high background suppression, spectra were measured from the recoiled evaporation residues (ER), from the $\alpha$ decay of those detector implanted neutron-deficient isotopes of elements from $\mathrm{Pt}$ to $\mathrm{Hg}$, and from $K-X$-rays emitted from nuclei after electron capture (EC).

These most successful test experiments led immediately to a search for SHN following Sikkeland's prescription. The reaction studied was ${ }^{136} \mathrm{Xe}+{ }^{170} \mathrm{Er} \rightarrow{ }^{306} 120 *$ in 1976 [21] and ${ }^{65} \mathrm{Cu}+{ }^{238} \mathrm{U} \rightarrow{ }^{303} 121^{*}$ [22] in 1977. Although the beam times were short, only one and two days were allocated, respectively, cross-section limits of about $1 \mathrm{nb}$ were reached. The promised island of SHN was not found, and technical improvements became necessary.

\section{Technical improvements and new results on synthesis and decay}

Relatively simple detector devices had been used in the early experiments. These were a $25 \mathrm{~mm}$ diameter multi-foil carbon secondary electron detector for a rough $Z$ determination described in [23], a commercial $450 \mathrm{~mm}^{2} \mathrm{Si}$ detector, and a single crystal Ge detector. Measurement of the distribution of reactions products in the focal plane of SHIP revealed that larger area detectors are needed for fully exploiting the separation properties of SHIP.

A first version of position sensitive Si detectors was developed in 1978 and used for experiments. Combining seven single detectors a total area of $87 \mathrm{~mm} \times 27 \mathrm{~mm}$ was covered in the focal plane. Two single foil secondary electron TOF detectors of $60 \mathrm{~mm} \times 30 \mathrm{~mm}$ [24] and a Ge detector completed the set-up. The new detector system was tested with the study of neutron-deficient $\alpha$ emitters along $N=82$. A number of new isotopes and $\alpha$ emitting yrast 


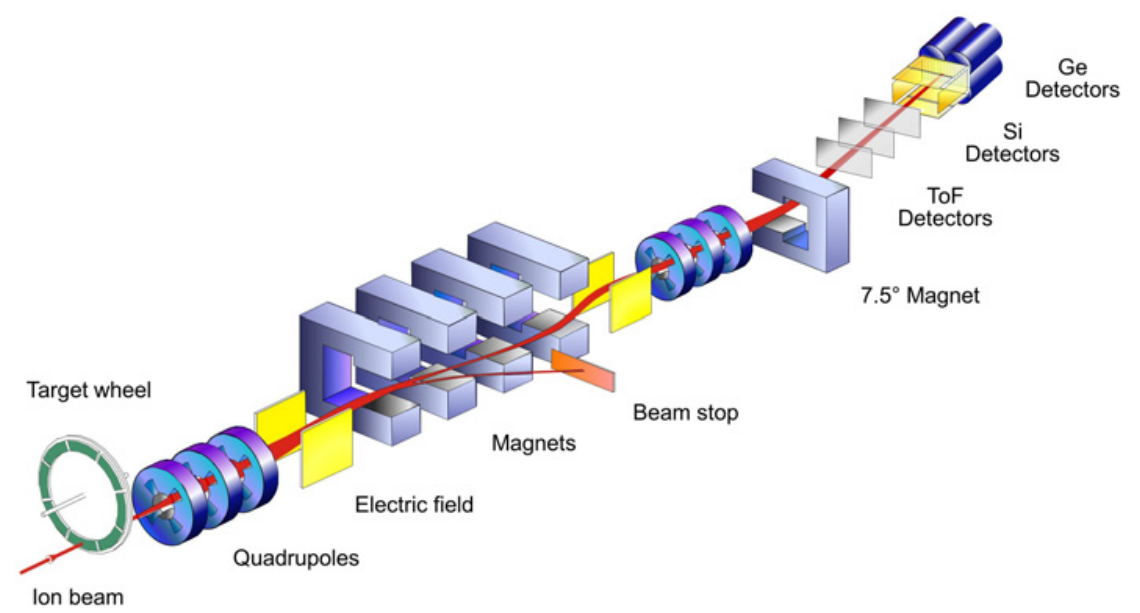

Figure 1. The velocity filter SHIP (Separator for Heavy Ion reaction Products) and its detection system [19, 25, 29]. The figure shows the upgraded version as it has been used since 1994. The drawing is approximately to scale, however, the target wheel and the detectors are enlarged by a factor of two. The length of SHIP from the target to the detector is $11 \mathrm{~m}$. The target wheel has a radius up to the center of the targets of $155 \mathrm{~mm}$. It rotates synchronously with beam macrostructure at $1125 \mathrm{rpm}$ [30]. The target thickness is usually $450 \mu \mathrm{g} / \mathrm{cm}^{2}$. The detector system consists of three large area secondaryelectron time-of-flight detectors [31], a position-sensitive silicon-detector array, and a germanium clover detector. The flight time of the reaction products through SHIP is $1-2 \mu$ s. The filter, originally consisting of two electric and four magnetic dipole fields plus two quadrupole triplets, was later extended by a fifth deflection magnet, allowing for positioning of the detectors away from the straight beam line and leading to further reduction of the background.

traps with half-lives below milliseconds were identified $[25,26]$. Of particular interest was the detection of the first proton-emitting isotope beyond the proton drip-line, ${ }^{151} \mathrm{Lu}$ [27].

Properties of fusion reactions were studied by K.H. Schmidt et al. [23, 28]. Neutrondeficient nuclei along $N=126$ were explored. New neutron-deficient isotopes and isomers of Pa were discovered in reactions of a ${ }^{40} \mathrm{Ar}$ beam and targets of ${ }^{181} \mathrm{Ta}$ and ${ }^{184} \mathrm{~W}$ [23]. Fusion hindrance was studied using symmetric systems [28].

Appropriate for the tracking of the lifetime of a single atom from the implantation into the detector up to its decay, the new method of plotting the counts on an axis at logarithmically increasing width of bins was introduced [23]. The center of gravity of the so determined universal decay curve marks the lifetime of the isotope. The significance of data in the case of poor statistics was investigated and procedures for determining error bars for lifetime and cross-section at poor statistics were elaborated [32].

Production of nuclei in the region of neutron deficient nuclei along the closed shell $N=126$ was considered exemplary for the synthesis of spherical SHN. In the focus of the interest was the damping of shell effects at high excitation energies. Systematic data for crosssections of ERs revealed no local increase from the continuously decreasing values when $N=126$ was crossed into direction of lighter isotopes. It was concluded that the damping of shell effects at high excitation energy will appreciably reduce the cross-section for production of SHN $[32,33]$.

Applying the improved techniques in the region of heavy elements, the first result of considerable importance was the identification of ${ }^{257} 104$ in the reaction ${ }^{50} \mathrm{Ti}+{ }^{208} \mathrm{~Pb} \rightarrow$ ${ }^{258} 104 *$ [34]. This was the first observation of the emission of only one neutron from the 
$\mathrm{CN}$ (1 $n$ channel) and a confirmation of results obtained at Dubna that cold fusion based on $\mathrm{Pb}$ or $\mathrm{Bi}$ targets provides a possibility for synthesis of heavy nuclei [35]. The also measured half-life of the spontaneously fissioning even-even nucleus ${ }^{256} 104$ produced in the $2 n$ channel confirmed the observation by Yu.Ts. Oganessian et al. [35] that the systematics of long half-lives of $N=152$ isotones is broken at element 104. This phenomenon was successfully explained using Strutinsky's method for calculation of fission barriers. It was shown that at high $Z$ the decreasing liquid drop fission barrier results in a depression of the second hump of the fission barrier below the ground-state, and the shorter SF half-lives result from a narrow single humped barrier [36].

The first new isotopes produced and identified at SHIP in the region of heavy elements were ${ }^{239} \mathrm{Cf}$ as $\alpha$-decay daughter of ${ }^{243} \mathrm{Fm}$ and ${ }^{247} \mathrm{Md}$ [37]. These nuclei were produced in reactions with a ${ }^{40} \mathrm{Ar}$ beam and targets of ${ }^{206} \mathrm{~Pb}$ and ${ }^{209} \mathrm{Bi}$, respectively.

\section{The new elements 107 to 109}

Element 107 was the first new element produced and identified at SHIP [38]. The reaction ${ }^{54} \mathrm{Cr}+{ }^{209} \mathrm{Bi} \rightarrow{ }^{263} 107^{*}$ was selected. Two reasons were decisive for that choice. Firstly, reactions with actinide targets were excluded, because beams lighter than ${ }^{40} \mathrm{Ar}$ were forbidden at GSI. There were several accelerators providing beams of light ions in Germany, and it was concluded that GSI should concentrate on reactions with heavy ions. Unfortunately, this decision prevented gaining experience with hot fusion, reactions based on actinide targets and beams of light ions, an experience that would have been very valuable later. Secondly, the preparatory experiments using $\mathrm{Pb}$ and $\mathrm{Bi}$ targets had delivered promising results in various test reactions before. The circumstance that these targets could be manufactured and handled without radiation protection was another supporting argument.

The irradiations for element 107 were performed in February 1981. The main experiment was preceded by a study of the decay of the expected $\alpha$-decay daughters, isotopes of elements 105 and $\mathrm{Lr}$, which were not known at that time. For that study a beam of ${ }^{50} \mathrm{Ti}$ ions was used. For the main experiment, beam time with a ${ }^{54} \mathrm{Cr}$ beam of only five days was allocated. Not without surprise, six decay chains were observed, five of them starting with $\alpha$ decays of $10.38 \mathrm{MeV}$ at lifetimes of $\tau=6.8 \mathrm{~ms}$ and one chain starting with $9.70 \mathrm{MeV}$ at $\tau=165 \mathrm{~ms}$. This chain and the longest one of the other five is plotted in Fig. 2. The 9.70-MeV decay was assigned to the decay of the ground state of ${ }^{262} 107$ and the higher energy decay to an isomeric state. The daughter decays were in agreement with the previously identified isotopes ${ }^{258} 105$ and ${ }^{254} \mathrm{Lr}$.

Changing the beam from ${ }^{54} \mathrm{Cr}$ to ${ }^{58} \mathrm{Fe}$ would produce element 109 . After emission of one neutron from the $\mathrm{CN}$ the produced isotope ${ }^{266} 109$ was expected to decay into the now known isotope ${ }^{262} 107$. The irradiation was performed in August 1982. In a three-days preparatory experiment the reaction ${ }^{50} \mathrm{Ti}+{ }^{209} \mathrm{Bi}$ was studied in more detail and a $22 \% \mathrm{EC}$ branch was determined for ${ }^{258} 105$. The main experiment lasted two weeks. Only one significant decay chain was measured starting with an $\alpha$ decay of $11.10 \mathrm{MeV}[39,40]$, see Fig. 2. The daughter decays were in agreement with known decays down to the spontaneously fissioning nucleus ${ }^{258} 104$.

Attempts to produce element 108 had been postponed. Calculations of SF half-lives of even- $N$ isotopes of element 108 resulted in values close to $1 \mu \mathrm{s}$ [36]. In that case the produced nuclei would have decayed before they reached the detector. However, it was not only the short half-life which determined the planning of experiments. Also in the case of a partial SF half-life shorter than the $\alpha$ half-life, an identification of the dominantly fissioning nucleus would be hampered. The already well established $\alpha-\alpha$ correlation method could not be applied 

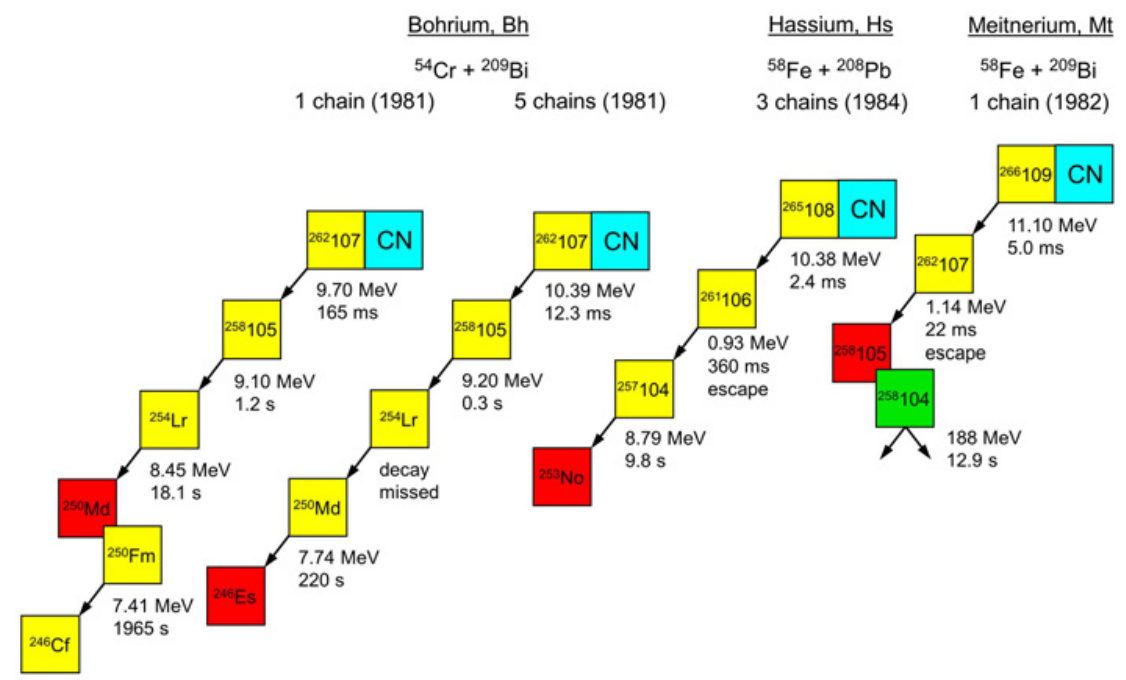

Figure 2. Examples of $\alpha$-decay chains measured in the search experiments for elements 107, 108, and 109. The first information from the decay chain was obtained from the implantation of the evaporation residue (ER) produced after emission of one neutron from the compound nucleus ( $\mathrm{CN}$, blue). For each $\alpha$-decaying nucleus (yellow) the measured $\alpha$ energy and the lifetime is given. The chains terminate by a known, but unobserved electron capture decay (EC, red), by the unobserved $\alpha$ decay of ${ }^{246} \mathrm{Cf}$ $\left(T_{1 / 2}=36 \mathrm{~h}\right)$ or, as in the case of ${ }^{266} 109$, by spontaneous fission (SF, green) after EC. The rows on top show name and symbol of the new element, beam and target of the fusion reaction, number of measured decay chains, and year of the experiment.

in that case. However, after measurement of $\alpha$ decay of ${ }^{266} 109$ it was expected that also isotopes of element 108 would preferably decay by $\alpha$ emission.

This assumption was corroborated in 1984, when in a preparatory experiment $\alpha$ decay of the isotopes ${ }^{259} 106$ and ${ }^{261} 106$ was measured and even for the even-even isotope ${ }^{260} 106$ the partial SF half-life did not decrease relative to that of the $\alpha$-decay daughter ${ }^{256} 104$ [41]. The measured total half-life of ${ }^{260} 106$ was $3.6 \mathrm{~ms}$ and a $1 / 1$ branching ratio $b_{\alpha} / b_{S F}$ was determined. The reaction for studying element 106 was ${ }^{54} \mathrm{Cr}+{ }^{208} \mathrm{~Pb}$.

It was expected that the reaction ${ }^{58} \mathrm{Fe}+{ }^{208} \mathrm{~Pb}$ would produce ${ }^{265} 108$ in a $1 n$-evaporation channel. Three decay chains of this isotope were measured in March 1984 [42]. The first one of these chains is shown in Fig. 2. The mean half-life was $1.8 \mathrm{~ms}$.

Two years later, the even-even nucleus ${ }^{264} 108$ was identified in a reaction of ${ }^{58} \mathrm{Fe}$ with a ${ }^{207} \mathrm{~Pb}$ target [43]. Only one decay chain was observed. Again, $\alpha$ decay was measured but the half-life was now $76 \mu \mathrm{s}$.

\section{Intermediate conclusions}

The realization of the technical concept of UNILAC and SHIP and subsequent development of position sensitive Si detectors has proven to be the right choice for study of rare nuclei and decay modes, in particular those of heavy nuclei. Short separation time, high background suppression and the use of a high-resolution detector system together with fast and low noise signal processing resulted in the identification of three new elements. Admittedly, the fact that both the decay of the nuclei by $\alpha$ emission resulting in decay chains with half-lives between milliseconds and some tens of seconds were within the window of optimal performance of the experimental set-up, was a lucky coincidence. 

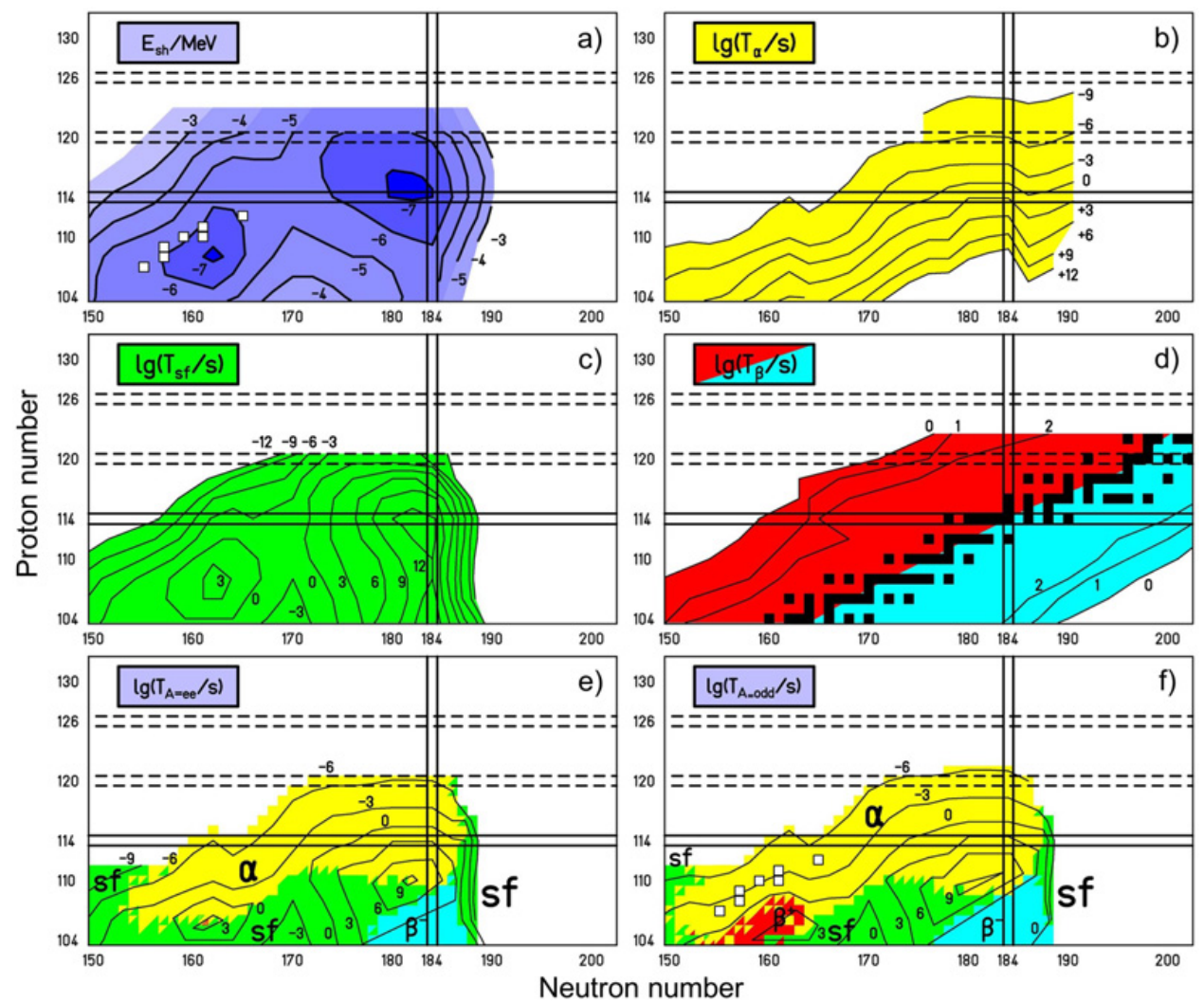

Figure 3. Shell-correction energy (a) and partial half-lives for $\alpha$ decay, SF, and $\beta$ decay (b)-(d). The calculated values in (a)-(c) were taken from [44, 45] and in (d) from [46]. The filled squares in (d) mark the $\beta$ stable nuclei. Half-lives and dominating decay modes for $\alpha, \beta^{+}$decay/EC, $\beta^{-}$decay, and SF are plotted for even-even nuclei in (e) and for odd-A nuclei in (f). Hindrance factors of 10 and 1000 were applied for $\alpha$ decay and SF of odd-A nuclei, respectively. White squares in (a) and (f) mark the produced nuclei ${ }^{262} 107,{ }^{265} 108,{ }^{266} 109,{ }^{269} 110,{ }^{271} 110,{ }^{272} 111$, and ${ }^{277} 112$ of the new elements from 107 to 112 .

On the other hand, the aim of the study was to discover the unknown properties of nuclei of new elements not knowing what the result would be. The now available experimental data could be compared with existing theoretical models. It turned out that the measured data could be well explained by the results of calculations by S.G. Nilsson et al. [8] already published in 1969 using the MM model. In those calculations, minimal negative SCE were calculated not only for spherical SHN but also for nuclei around $Z=108$ and $N=162$. However, there, in contrast to the spherical SHN, the increased stability occurs at relatively large quadrupole deformation due to a relatively high level density for protons and neutrons below level gaps at $Z=108$ and $N=162$, respectively.

Later, more refined calculations presented in [46-48] substantiated the early results. SCE values, partial half-lives, and deduced dominant decay modes from these MM model calculations are shown in Fig. 3. In Fig. 3a the two regions of minimal SCE for deformed and spherical nuclei are clearly visible. Both regions are separated by a ridge of weak SCE at about mass number $A=280$, which separates regions of heavy and super-heavy nuclei. The produced nuclei of the new elements from 107 to 109 and those later identified from elements 110 to 112 marked in Figs. 3a are crossing the region of deformed nuclei. Their 
dominant decay mode is $\alpha$ emission with half-lives from 0.1 to $100 \mathrm{~ms}$, well in agreement with the results of the theoretical models, see Fig. $3 \mathrm{f}$.

\section{The new elements 110 to 112}

Measured $1 n$ cross-sections for the production of elements from 102 to 109 in reactions with ${ }^{208} \mathrm{~Pb}$ and ${ }^{209} \mathrm{Bi}$ revealed an average decrease by a factor of 4.4 per element. An extrapolation to element 110 resulted in a cross-section of $1.5 \mathrm{pb}$. In contrast to the cross-sections of these so called cold fusion reactions, the extrapolation of known cross-sections of the hot fusion reactions for elements from 102 to 106 based on targets from ${ }^{238} \mathrm{U}$ to ${ }^{249} \mathrm{Cf}$ was worse. A cross-section of $0.1 \mathrm{pb}$ was extrapolated for element 110 . For production of one atom of element 109 at a cross-section of $16 \mathrm{pb}$ we needed two weeks of beam time. Under the same conditions, we estimated needing 20 weeks for production of element 110, not taking into account statistical fluctuations.

Such a long beam time could not be allocated at the end of the 1980s. The new synchrotron SIS had been built at GSI and other experiments became the center of interest. In addition, predictions of the reaction theory were not promising. Support for the GSI heavy element program was reduced. Under these circumstances we were forced to improve the experimental set up. The aim was to reach a cross-section of $1 \mathrm{pb}$ in two weeks of beam time.

The improvements were performed during the years 1988 to 1994 . The largest gain factor of 2.9 was due to installation of a new high charge-state injector based on an ECR ion source and a 108-MHz RFQ-IH injection accelerator for the Alvarez section of the UNILAC. In this case we profited from the request of other experiments for a stable uranium beam. The injector delivered higher beam intensities at higher stability, an increase of the length of beam pulses to $5.5 \mathrm{~ms}$, and considerably less consumption of isotopically enriched source material. Other improvements were related to an increase of the SHIP acceptance angle by moving the target closer to the first quadrupole, an optimization of the target thickness and new, larger area TOF and position sensitive Si detectors. A fifth deflection magnet reduced the background considerably. The upgraded version of SHIP is shown in Fig. 1.

A serious problem which had to be solved experimentally before starting the main experiment to search for element 110 was caused by reaction theory. It was predicted that with heavier $Z$ projectiles increasing beam energies are needed for fusion of nuclei of high proton number. An extra-push of $30 \mathrm{MeV}$ calculated for the reaction $\mathrm{Ni}+\mathrm{Pb}$ in [49] belonged to the smaller values predicted. Such an increase of the resulting excitation energy $E^{*}$ would have reduced the survival probability of the $\mathrm{CN}$ considerably.

The quality of excitation functions measured for element 102 to 107 in cold fusion reactions was insufficient for extracting an extra-push energy reliably and thus determining an optimum beam energy solely based on experimental data. In addition, element 108 and 109 were observed only at one relatively high energy which was chosen taking into account a certain amount of extra-push energy.

In order to obtain a reliable value for the optimum beam energy for production of element 110 , we decided to measure accurately the excitation functions for the reactions ${ }^{50} \mathrm{Ti}+{ }^{208} \mathrm{~Pb}$ and ${ }^{58} \mathrm{Fe}+{ }^{208} \mathrm{~Pb}$. The first measurement should be relatively fast due to the high crosssection. The second was necessary because only one energy of the excitation function was known. In addition, element 108 was the closest approach of an even element to the even element 110. However, for this latter measurement an additional problem arose from the fact, that we needed an amount of approximately $4 \mathrm{~g}$ of enriched ${ }^{58} \mathrm{Fe}$, which cost at that time $500 \mathrm{kDM} / \mathrm{g}$. The necessary amount of money could not be provided by GSI. Therefore, we were extremely happy that our collaborators from the Flerov Laboratory of Nuclear Reactions 


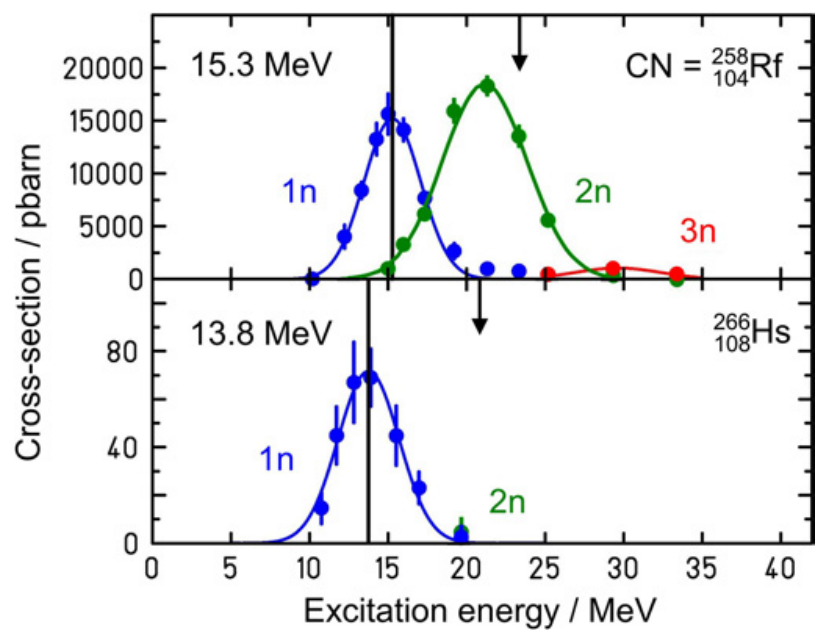

Figure 4. Measured excitation functions for production of isotopes of elements 104 (Rf) and 108 (Hs) by reactions of ${ }^{50} \mathrm{Ti}$ and ${ }^{58} \mathrm{Fe}$ beams with ${ }^{208} \mathrm{~Pb}$ targets. The cross-sections are plotted as a function of the excitation energy, $E^{*}$, calculated from the beam energy in the middle of the target thickness. The curves represent Gaussian curves fitted to the data points. An extrapolation of the maxima for element 104 and 108 resulted in an optimum value of $E^{*}=13.0 \mathrm{MeV}$ for production of element 110 . The arrows mark the interaction barriers of the reactions according to the fusion model of R. Bass [50]. These barriers are defined by a beam energy so that beam and target nuclei are at rest in the center of mass system when their mean radii are at contact.

(FLNR) in Dubna contributed the material. Later, we were able to reimburse part of the efforts by supporting our collaborators at FLNR with detectors and electronics.

The excitation function for element 104 was measured in June, that of 108 in October 1994. The measured data are shown in Fig. 4. Now it was easy to extrapolate the well determined cross-section maxima of the $1 n$ curves to element 110 , and already on the first day of the irradiation of a ${ }^{208} \mathrm{~Pb}$ target with ${ }^{62} \mathrm{Ni}$ ions the first decay chain of ${ }^{269} 110$ was measured [51]. The experiment lasted twelve days, and a total of four decay chains was accumulated. The longest one consisting of five consecutive $\alpha$ decays is shown in Fig. 5. The isotope ${ }^{62} \mathrm{Ni}$ was chosen with the assumption that the addition of only one $\alpha$ particle to the ${ }^{58} \mathrm{Fe}$ beam used for element 108 would be a minimal change of the reaction and, secondly, the new isotope ${ }^{269} 110$ could be unambiguously identified by the then well known decay properties of its daughter ${ }^{265} 108$.

In a second step, the most neutron rich stable $\mathrm{Ni}$ isotope, ${ }^{64} \mathrm{Ni}$, was used as a beam. The yield increased and during another thirteen days a total of nine decay chains was measured of ${ }^{271} 110$ [52]. One of the two longest chains is shown in Fig. 5. The majority of eight chains started with a half-life of $1.1 \mathrm{~ms}$ of ${ }^{271} 110$, in one case a half-life of $56 \mathrm{~ms}$ was measured. Using the argument that high-spin nuclei are populated stronger in fusion reactions, it seems reasonable to assign the eight chains to the decay of a high-spin state which decays preferably into a high-spin state of the daughter nucleus whereas the decay with the long half-life is attributed to a low-spin state, which, however, is hindered by a change of angular momentum. High- and low-spin states near the ground-state of ${ }^{271} 110$ and its daughter ${ }^{267} 108$ were obtained in a theoretical work of S. Cwiok et al. [53]. The suggested decay scheme is presented in [52]. 


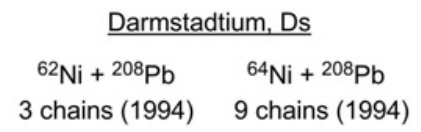

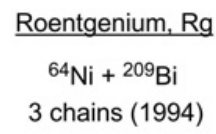

\section{Copernicium, $\mathrm{Cn}$ \\ ${ }^{70} \mathrm{Zn}+{ }^{208} \mathrm{~Pb}$ \\ 1 chain (1996)}

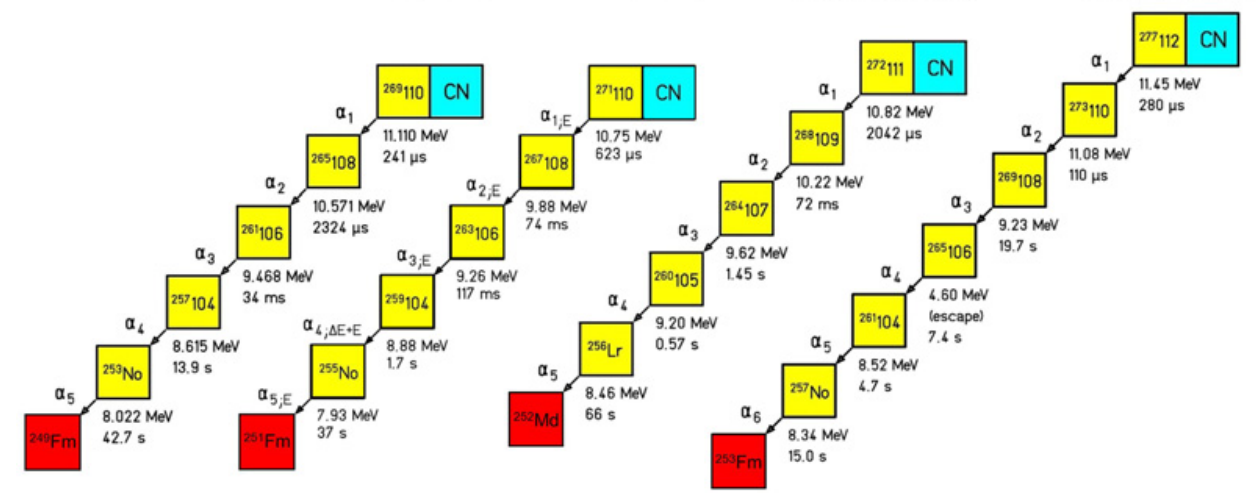

Figure 5. Examples of $\alpha$-decay chains measured in the search experiments for elements 110, 111, and 112. The first information from the decay chain was obtained from the implantation of the evaporation residue (ER) produced after emission of one neutron from the compound nucleus ( $\mathrm{CN}$, blue). For each $\alpha$-decaying nucleus (yellow) the measured $\alpha$ energy and the lifetime is given. The chains terminate by a known, but unobserved electron capture decay (EC, red). The rows on top show name and symbol of the new element, beam and target of the fusion reaction, number of measured decay chains, and year of the experiment.

Eventually, the target was changed to ${ }^{209} \mathrm{Bi}$ aiming to search for element 111 . During an irradiation period of seventeen days we measured three decay chains of ${ }^{272} 111$ in December 1994 [54]. The longest of the chains is shown in Fig. 5.

Element 112 was searched for using the most neutron-rich stable isotope of $\mathrm{Zn},{ }^{70} \mathrm{Zn}$, and a ${ }^{208} \mathrm{~Pb}$ target in January-February 1996 [55]. Two decay chains were published, the second and longest one is shown in Fig. 5. Already in the publication [55] it was mentioned that the second chain was more complete than the first one. In a confirmation experiment performed in 2000 a third chain was measured, which was in agreement with the second chain down to ${ }^{265} \mathrm{Sg}$, where the new chain ended by a previously unknown SF branch [56]. A reanalysis of the 1996 data in connection with the confirmation experiment revealed that the first chain published in 1996 did not exist in the original data. This chain had to be retracted [56].

Because of this, reanalysis of all the data on elements 110, 111, and 112 measured at GSI since 1994 logically followed (a total of 34 decay chains was investigated) and revealed that the second chain of ${ }^{269} 110$ was not recorded in the original data files in 1994 . This chain was also retracted [56]. In all other cases the earlier data were exactly reproduced in the reanalysis.

Four further experiments using cold fusion were negative. Nevertheless, they are mentioned here because also the obtained cross-sections limits were of importance.

Firstly, the systematics of $1 n$ cross-section maxima of cold fusion reactions revealed a continuous trend to lower excitation energy with increasing proton number of the beam, see Fig. 4 and Figs. 19 and 21 in [52]. Cross-section maxima were measured at beam energies just enough high so that beam and target nuclei come to rest in the center of mass system, when their diffused surfaces are touching. It was noticed that this configuration results in excitation energies below the $1 n$ binding energy for reactions with beams of ${ }^{76} \mathrm{Ge}$ and ${ }^{82} \mathrm{Se}$. Therefore, the possibility of a radiative capture process was tested using the reaction ${ }^{82} \mathrm{Se}+{ }^{208} \mathrm{~Pb}$ in 
1995. At four beam energies between $E^{*}=0$ and $10 \mathrm{MeV}$ cross-section limits of $5 \mathrm{pb}$ were reached [52]. A decay from a possible production of the nucleus ${ }^{290} 116$ was not observed.

Secondly, using the reaction ${ }^{68} \mathrm{Zn}+{ }^{208} \mathrm{~Pb}$ we tried to produce a lighter isotope of element 112. In that reaction the $\mathrm{CN}$ having $N=164$ is closer to the center of increased stability at $N=162$ than in the reaction with the ${ }^{70} \mathrm{Zn}$ beam, and a higher cross-section could result, similar to the case of element 110 when the beam was changed from ${ }^{62} \mathrm{Ni}$ to ${ }^{64} \mathrm{Ni}$, see Fig. 3(a). At two beam energies one-event cross-section limits of $0.7 \mathrm{pb}$ were obtained in 1997 comparable to that of $0.5 \mathrm{pb}$ measured with a ${ }^{70} \mathrm{Zn}$ beam. A strong increase of the crosssection due to shell effects at $N=162$ can be excluded. More importantly, the higher yield with the ${ }^{70} \mathrm{Zn}$ beam seems to be with the higher number of neutrons.

Thirdly, a search for element 113 started in 1998 and was continued in 2003. The reaction studied was ${ }^{70} \mathrm{Zn}+{ }^{209} \mathrm{Bi}$. During a total irradiation time of 57 days, a one-event cross-section limit of $160 \mathrm{fb}$ was reached [57]. The experiment had to be stopped due to lack of beam time. However, a successful irradiation was performed at RIKEN. Using the same reaction, three decay chains assigned to ${ }^{278} 113$ were measured during a total of 553 days of beam time accomplished in several parts between 2003 and 2012 [58]. The measured cross-section of $28 \mathrm{fb}$ is the smallest one ever achieved in a heavy-ion fusion reaction.

Fourthly, the reaction ${ }^{86} \mathrm{Kr}+{ }^{208} \mathrm{~Pb}$ was studied subsequently to reports on positive results of the synthesis of element 118 in 1999 [59]. A one-event cross-section limit of $0.5 \mathrm{pb}$ was reached at SHIP in 24 days of beam time. Although lower than the Berkeley value of $2.2 \mathrm{pb}$ published for three decay chains, our limit did not exclude the correctness of the Berkeley result. Eventually, the first announcement was retracted in 2001 after additional experiments in Berkeley and after a reanalysis of the data of the first experiment [60].

\section{Concluding remarks}

The half-lives and decay modes of the produced nuclei of the new elements from 107 to 112 are well in agreement with the results of the MM model. Shell effects increase the stability of nuclei around $Z=108$ and $N=162$. However, nuclei in that region are deformed in contrast to the spherical SHN. Although minimal SCE values are at $-7 \mathrm{MeV}$ in both regions, see Fig. 3a, the calculated partial SF half-lives are considerably shorter in the region of deformed nuclei, $10^{3} \mathrm{~s}$ instead of $10^{12} \mathrm{~s}$, see Fig. $3 \mathrm{c}$. The more narrow fission barrier and the already elongated shape of the deformed nuclei is responsible for the difference.

Measured $\alpha$ half-lives, although less influenced by shell effects, prove that the region of highest stability is already surpassed with ${ }^{277} 112$. High $\alpha$ energies and accordingly short half-lives are measured for the first two decays of the chain, which proceed into direction of increased stability, see Fig. 3. Energies decrease by $2 \mathrm{MeV}$ and half-lives increase by almost five orders of magnitude for the decay of ${ }^{269} 108$, see Fig. 5, when the region of highest stability is left into the direction of smaller proton and neutron numbers.

The low cross-section for production of element 113 and the negative results of the search experiments for elements 116 and 118 by cold fusion is understandable by means of Fig. 3 a. In addition to increasing re-separation probability of beam and target fragments due to higher proton number of the beam, the locations of the $\mathrm{CN}$ are departing the region of strong shell effects and thus high fission barriers of deformed nuclei, but, for the heaviest systems, the $\mathrm{CN}$ are not located within the region of strong shell effects of spherical SHN.

At the end of the 1990s, the technical requirements for continuing the experiments at GSI and SHIP using hot fusion reactions were excellent. An intensive and stable beam of ${ }^{48} \mathrm{Ca}$ was achievable from the ECR ion source. The experiments on identification of new elements using cold fusion have convincingly demonstrated the high efficiency and background suppression 
of the separator SHIP and the reliable identification of the produced nuclei by the positiontime correlation method.

However, rather pessimistic perspectives were published for a production of SHN by hot fusion reactions $[61,62]$. Nevertheless, when we developed a medium-range plan for experiments at SHIP at the end of 1998, which was requested by the GSI management, we also suggested to study hot fusion reactions based on ${ }^{48} \mathrm{Ca}$ beams for synthesis of SHN, knowing that a larger program for such an investigation was already started at FLNR in Dubna. In our proposal we also attempted to stimulate a discussion on the option of a new accelerator delivering high intensity DC beams dedicated to low-energy experiments, in particular to research on SHN. This happened at the time, when an extension of the GSI accelerator facilities were discussed, aiming at higher currents at relativistic energies. With our proposal we hoped to prepare a bright future for SHN research at GSI. We attempted to make available our suggestions to the public in form of a report [63]. However, already at the beginning of 1999, we were forced to retract that report. Distribution and thus a public discussion was prohibited. The study of hot fusion reactions at SHIP had to be postponed.

The decisive experiments on exploration of the island of spherical SHN were performed at FLNR in Dubna. During the years 1999 to 2010 nuclei of the new elements 113 to 118 were produced and identified [64]. Cross-sections increased with approach of the $\mathrm{CN}$ to the center of the island of stability, see Fig. 3a. Values of $10 \mathrm{pb}$ were measured for production of isotopes of elements 114 and 115 in reactions of a ${ }^{48} \mathrm{Ca}$ beam with targets of ${ }^{244} \mathrm{Pu}$ and ${ }^{243} \mathrm{Am}$, respectively. Decay modes, $\alpha$-decay half-lives and in particular the systematics of partial SF half-lives confirmed the existence of a region of increased stability due to a double shell closure at $Z=114$ and $N=184$, as it was predicted already in the 1960 s by theoretical models.

The excellent performance of SHIP also for the study of nuclei produced in hot fusion reactions was demonstrated later when targets of ${ }^{238} \mathrm{U}$ and ${ }^{248} \mathrm{Cm}$ were irradiated with ${ }^{48} \mathrm{Ca}$ ions $[65,66]$. Results, previously obtained at FLNR, were confirmed, and energies of the $\alpha$ decays were improved. In a continuation of the experiments based on a ${ }^{248} \mathrm{Cm}$ target we attempted to produce element 120 using a beam of ${ }^{54} \mathrm{Cr}$. The results of this search are presented in a recent review article [67], and a related study on fission barriers deduced from $\alpha$-decay chains is published in [68]. Experiments to search for element 120 will be continued at FLNR [69] and at RIKEN [70]. At GSI, the UNILAC which was so important for a successful low-energy program and in particular the study of SHN, is presently optimized for injection of short beam pulses into the accelerators of the new Facility for Antiproton and Ion Research (FAIR). It will not be available for a heavy element program in the future.

\section{References}

[1] M. Goeppert Mayer, Nobel Lecture: The Shell Model, http://www.nobelprize. org/nobel_prizes/physics/laureates/1963/mayer-lecture.html.

[2] V.M. Strutinsky, Nucl. Phys. A95, 420 (1967).

[3] W.D. Myers and W.J. Świątecki, Nucl. Phys. 81, 1 (1966).

[4] A. Sobiczewski, F.A. Gareev, B.N. Kalinkin, Phys. Lett. 22, 500 (1966).

[5] H. Meldner, Ark. Fys. 36, 593 (1967).

[6] S.G. Nilsson et al., Nucl. Phys. A115, 545 (1968).

[7] S.G. Nilsson, S.G. Thompson, C.F. Tsang, Phys. Lett. B28, 458 (1969).

[8] S.G. Nilsson et al., Nucl. Phys. A131, 1 (1969).

[9] U. Mosel, W. Greiner, Z. Phys. 222, 261 (1969).

[10] J. Grumann et al., Z. Phys. 228, 371 (1969). 
[11] E.O. Fiset and J.R. Nix, Nucl. Phys. A193, 647 (1972).

[12] A. Sobiczewski, Physica Scripta A 10, 47 (1974).

[13] J. Randrup et al., Phys. Rev. C 13, 229 (1976).

[14] S.M. Polikanov et al., Sov. Phys. JETP 15, 1016 (1962).

[15] T. Sikkeland, Ark. Fys. 36, 539 (1967).

[16] G.N. Flerov et al., JINR P7-3808 (1968).

[17] A. Ghiorso et al., Phys. Rev. Lett. 24, 1498 (1970).

[18] F. Beck et al., Memorandum zur Errichtung eines gemeinsamen Ausbildungszentrum für Kernphysik Hessischer Hochschulen Darmstadt, Frankfurt und Marburg (1966).

[19] G. Münzenberg et al., Nucl. Instr. Meth. 161, 65 (1979).

[20] P. Armbruster, Proc. 3rd Int. Conf. on Nuclei far from Stability, Report CERN 76-13, 3 (1976).

[21] P. Armbruster et al., GSI Jahresbericht 1976, GSI Report GSI-J-1-77, 74 (1977).

[22] G. Münzenberg et al., GSI Jahresbericht 1977, GSI Report GSI-J-1-78, 75 (1978).

[23] K.H. Schmidt et al., Nucl. Phys. A318, 253 (1979).

[24] F.P. Heßberger et al., GSI Scientific Report 1981, GSI Report GSI-82-1, 223 (1982).

[25] S. Hofmann et al., Z. Phys. A291, 53 (1979).

[26] S. Hofmann et al., Nucl. Instr. Meth. 223, 312 (1984).

[27] S. Hofmann et al., Z. Phys. A305, 111 (1982).

[28] C.C. Sahm et al., Z. Phys. A319, 113 (1984).

[29] S. Hofmann, G. Münzenberg, Rev. Mod. Phys. 72, 733 (2000).

[30] H. Folger et al., Nucl. Instr. Meth. A362, 64 (1995).

[31] S. Saro et al., Nucl. Instr. Meth. A381, 520 (1996).

[32] K.H. Schmidt et al., Z. Phys. A316, 19 (1984).

[33] K.H. Schmidt, J.G. Keller, D. Vermeulen, Z. Phys. A315, 159 (1984).

[34] G. Münzenberg et al., GSI Scientific Report 1979, GSI Report GSI-80-3, 59 (1980).

[35] Yu.Ts. Oganessian et al., Nucl. Phys. A239, 157 (1975).

[36] A. Baran et al., Proc. 3rd Int. Conf. on Nuclei far from Stability, Report CERN 76-13, 537 (1976).

[37] G. Münzenberg et al., Z. Phys. A302, 7 (1981).

[38] G. Münzenberg et al., Z. Phys. A300, 107 (1981).

[39] G. Münzenberg et al., Z. Phys. A309, 89 (1982).

[40] G. Münzenberg et al., Z. Phys. A315, 145 (1984).

[41] G. Münzenberg et al., Z. Phys. A322, 227 (1985).

[42] G. Münzenberg et al., Z. Phys. A317, 235 (1984).

[43] G. Münzenberg et al., Z. Phys. A324, 489 (1986).

[44] R. Smolanczuk, J. Skalski, A. Sobiczewski, Phys. Rev. C 52, 1871 (1995).

[45] R. Smolanczuk and A. Sobiczewski, Proc. XV. Nuclear Physics Divisional Conference on Low Energy Nuclear Dynamics, St.Petersburg, Russia, 1995, World Scientific, Singapore, p. 313 (1995).

[46] P. Möller, J.R. Nix, K.L. Kratz, At. Data Nucl. Data Tables 66, 131 (1997).

[47] A. Sobiczewski and K. Pomorski, Prog. Part. Nucl. Phys. 58, 292 (2007).

[48] P. Möller et al., At. Data Nucl. Data Tables 59, 185 (1995).

[49] P. Fröbrich, Phys. Lett. B215, 36 (1988).

[50] R. Bass, Nucl. Phys. A231, 45 (1974).

[51] S. Hofmann et al., Z. Phys. A350, 277 (1995).

[52] S. Hofmann, Rep. Prog. Phys. 61, 639 (1998).

[53] S. Cwiok, S. Hofmann, W. Nazarewicz, Nucl. Phys. A573, 356 (1994).

[54] S. Hofmann et al., Z. Phys. A350, 281 (1995). 
[55] S. Hofmann et al., Z. Phys. A354, 229 (1996).

[56] S. Hofmann et al., Eur. Phys. J. A14, 147 (2002).

[57] S. Hofmann et al., GSI Scientific Report 2003, GSI Report GSI-2004-1, 1 (1980).

[58] K. Morita et al., J. Phys. Soc. Jpn. 81, 103201 (2012).

[59] V. Ninov et al., Phys. Rev. Lett. 83, 1104 (1999).

[60] V. Ninov et al., Phys. Rev. Lett. 89, 039901 (2002).

[61] K.H. Schmidt and W. Morawek, Rep. Prog. Phys. 54, 949 (1991).

[62] P. Armbruster, Rep. Prog. Phys. 62, 465 (1999).

[63] S. Hofmann, SHIP-2000 - A proposal for the study of superheavy elements, GSIReport GSI-99-02, 1 (1999).

[64] Yu.Ts. Oganessian and V.K. Utyonkov, Nucl. Phys. A944, 62 (2015).

[65] S. Hofmann et al., Eur. Phys. J. A32, 251 (2007).

[66] S. Hofmann et al., Eur. Phys. J. A48, 62 (2012).

[67] S. Hofmann et al., Eur. Phys. J. A52, 180 (2016).

[68] S. Hofmann et al., Eur. Phys. J. A52, 116 (2016).

[69] V.K. Utyonkov, in Int. Symposium 'Super Heavy Nuclei', College Station, Texas, USA, 2015, http://cyclotron.tamu.edu/she2015/assets/pdfs/ presentations/Utyonkov_SHE_2015_TAMU.pdf .

[70] K. Morita, Int. Symposium 'Super Heavy Nuclei', College Station, Texas, USA, 2015, http://cyclotron.tamu.edu/she2015/assets/pdfs/

presentations/Morita_SHE_2015_TAMU.pdf. 